\title{
Differenz
}

Revista internacional de estudios heideggerianos y sus derivas contemporáneas

AÑO 7, NÚMERO 6: JULIO DE 2020. ISSN 2386-4877 - DOI: 10.12795/Differenz.2020.i06.05

[pp. 91-116]

Recibido: $30 / 04 / 2020$

Aceptado: 10/06/2020

\section{Nichilismo e linguaggio poetico nella riflessione "estetica" heideggeriana.}

\section{Nihilism and poetic language in Heideggerian "aesthetic" reflection.}

\author{
Danilo Serra \\ Università degli Studi di Bergamo
}

Sunto:

II seguente contributo vuole mostrare come la questione circa la natura della Dichtung permetta ad Heidegger da un lato di misurarsi fino in fondo con le fondamentali contraddizioni ed inquietudini della contemporaneità, dall'altro di ripensare (e ricreare) la relazione originaria dell'uomo con le cose stesse e con l'essere. Da qui la necessità di riflettere sulla portata ontologica del discorso poetico con il fine di soffermarsi sulla peculiarità di un linguaggio, quello artistico-poetico, in grado di dirci, più di ogni altro, qualcosa di significativo sull'essenza dei fenomeni e sul nostro rapporto con la natura.

Parole chiave: Arte; Poesia; Cosa; Natura; Tecnica. 


\section{Abstract:}

The following contribution wants to show how the question about the nature of Dichtung allows Heidegger on the one hand to fully measure himself against the fundamental contradictions and anxieties of the contemporary world, and on the other hand to rethink (and recreate) man's original relationship with things themselves and with being. Hence the need to reflect on the ontological scope of poetic discourse with the aim of dwelling on the peculiarity of a language, the artistic-poetic one, able to tell us, more than any other, something significant about the essence of phenomena and our relationship with nature.

Keywords: Art; Poetry; Thing; Nature; Technique.

\section{Introduzione.}

Alla base del progetto filosofico heideggeriano sta la riflessione sulla tesi dell'unità di soggetto e oggetto, una questione che Heidegger affronta decisamente fin dai suoi corsi universitari tenuti nel primo periodo friburghese ${ }^{1}$. L'intera narrazione metafisica, così come singolarmente intesa dal pensatore di Messkirch, ruota attorno alla problematica circa la contrapposizione tra un soggetto via via interpretato come hypokéimenon -e cioè letteralmente come quel che "giace al di sotto", il "sostrato" che permane e sorregge ogni rappresentazione e determinazione dell'ente- e un oggetto compreso come ciò che cade sotto il giogo soggettivo (cioè del soggetto) dello sfruttamento e della consumazione. Si tratta, dunque, di una narrazione che, mettendo l'uno contro l'altro il soggetto e l'oggetto, I'uomo e il mondo, sviluppa un vero e proprio impianto teorico in cui il secondo termine appare sempre condizionato e vincolato al primo. L'oggetto, in altri termini, è subordinato alla calcolante determinazione del soggetto, da cui per l'appunto dipende. Allontanandosi da questo schema interpretativo, attraverso l'elaborazione di un programma di ricerca denominato existenziale Analytik des Dasein Heidegger si preoccupa di riconsiderare l'antitesi soggetto-oggetto, confrontandosi esplicitamente con le concrete manifestazioni

1 Per una cronologia dei corsi universitari tenuti da Heidegger nel primo periodo friburghese si veda esemplarmente KISIEL, T. The Genesis of Heidegger's "Being and Time". Berkeley, University of California Press, 1993. Cfr. altresì FABRIS, A. "L'ermeneutica della fatticità' nei corsi friburghesi dal 1919 al 1923" in VolPI, F. (a cura di). Guida a Heidegger. Bari, Laterza, 1998. Un elenco completo, approvato dallo stesso Heidegger, dei corsi universitari da lui tenuti è pubblicato in RICHARDSON, W. J. Heidegger. Through Phenomenology to Thought. The Hague, Nijhoff, 1963, pp. 663-671. 
esistenziali dell'essere umano². Così, le nozioni di Dasein e di In-der-Welt-Sein consentono al filosofo di reinterpretare il problema della soggettività al fine di sviluppare una comprensione genuina e originaria dell'esistenza dell'uomo che tenga conto della coappartenenza e dell'inscindibilità di uomo e mondo. Il che significa altresì affermare una riflessione sull'esistenza umana che si spinga ben oltre l'analisi sulla natura umana condotta dal cartesianesimo (riattualizzazione dell'antica opposizione corpo-anima e nuova contrapposizione tra res extensa e res cogitans), in modo da riconsiderare l'esserci, il Dasein, quale unità e fenomeno unitario:

L'espressione composta "essere-nel-mondo" mostra già, nella sua stessa coniazione, che con essa si intende un fenomeno unitario. Questo reperto primario va visto nella sua totalità ${ }^{3}$.

Se, però, è vero che da un lato Sein und Zeit, specie attraverso una riproblematizzazione della soggettività, indiscutibilmente prende le distanze dal sentiero battuto dal pensiero metafisico, dall'altro lato è vero anche che quest'opera, in senso lato, non riesce a superare del tutto le radicate costrizioni metafisiche. Benché Heidegger si proponga inizialmente di affrontare la grande e plurisecolare questione dell'essere, egli alla fine è costretto a rimettere in discussione la sua analitica, obiettando contro un criterio metodologico effettivamente incapace di penetrare nel cuore della Seinsfrage. Nell'evoluzione del suo pensiero, ciò di cui egli si avvede è che cercare di raggiungere l'essere a partire dall'esserci (dall'analitica esistenziale) non è affatto possibile, bensì risulta assai fuorviante e pericoloso. II rischio è, infatti, quello di oggettivare l'essere e così ridurlo a mero oggetto di indagine ed analisi. Detto in alto modo, il rischio è quello di determinare l'essere sul modello dell'ente, riproponendo paradossalmente l'opposizione metafisica tra soggetto determinante ed oggetto determinato.

Ciò che manca in Sein und Zeit è proprio, per dirla con le parole del pensatore tedesco, un linguaggio capace di parlare dell'essere. Così, l'opera del 1927, per sua stessa costituzione, non è in grado, dopotutto, di volgere lo sguardo al di là di un'analisi impegnata ad esaminare i caratteri costitutivi dell'esserci e i modi in cui esso sta nel mondo. Tutto ciò si manifesta come un vero e proprio limite o fallimento che porta l'autore a ricercare immediatamente altri stili e linguaggi con l'obiettivo di muovere una riformulazione della questione dell'essere senza ricadere nella sunnominata opposizione.

2 Cfr. HeIDEgGeR, M. Sein und Zeit (1927). Gesamtausgabe (d'ora in avanti GA) Bd. 2. Frankfurt am Main, Klostermann, 1997, §§ 4-5, 9-11, 28, 39, 63, 66, 83 (trad. it. Marini, A. Essere e tempo. Milano, Mondadori, 2018).

3 HeIdegger M. Essere e tempo. Op. cit. p. 83. 
A questo riguardo, decisivo ed avvincente appare il confronto con la poesia di Hölderlin ${ }^{4}$. Detto confronto, difatti, offre ad Heidegger da un lato l'opportunità di riscattare l'insuccesso di Sein und Zeit, dall'altro la possibilità di svincolarsi ancor più chiaramente dal linguaggio concettuale della filosofia tradizionale ${ }^{5}$. Ragion per cui, come meglio vedremo nel corso della seguente ricerca, non è errato sostenere che il poeta sia uno dei punti di riferimento principali nella matura riflessione filosofica heideggeriana. In particolare, nel dialogo con la poesia di Hölderlin Heidegger ridefinisce e mette in gioco i temi fondanti del suo pensiero. Attraverso la ripresa dell'opera hölderliniana, egli giunge a ripensare il modo in cui studiare e comprendere il fenomeno e la sua datità, rompendo definitivamente con la tradizione metafisica, cominciando seriamente a fare i conti con la natura ontologica del linguaggio poetico. Il poeta diviene così imprescindibile per ridiscutere allo stesso tempo la natura della poesia e il significato originario del Denken. In questo modo, Heidegger sviluppa una modalità di pensiero che chiama Andenken ${ }^{6}$ e che contrappone a quello concettuale del "rappresentare" (Vorstellen) proprio della metafisica occidentale.

Se la tradizione metafisica non ha fatto altro che riproporre nel tempo l'uguaglianza tra pensare e rappresentare, secondo la quale pensare significa sostanzialmente "fare di qualcosa un oggetto, porlo come oggetto e rappresentarlo solo così" sua, fermamente sostiene la tesi secondo cui il pensiero non è mai, in fondo, pensiero

4 Heidegger si occupa della ricerca hölderliniana in più scritti e conferenze che oggi ritroviamo nel volume intitolato Erläuterungen zu Hölderlins Dichtung (1936-1968). GA 4. Frankfurt am Main, Klostermann, 1981 (trad. it. Amoroso, L. La poesia di Hölderlin. Milano, Adelphi, 2001). Al poeta svevo, il filosofo dedica inoltre tre corsi universitari: Hölderlins Hymnen "Germanien" und "Der Rhein" (WS 1934/35). GA 39. Frankfurt an Main, Klostermann, 1980 (trad. it. Demarta, G. B. Gli inni di Hölderlin "Germania" e "Il Reno". Milano, Bompiani, 2005); Hölderlins Hymne "Andenken" (WS 1941/42). GA 52. Frankfurt an Main, Klostermann, 1982 (trad. it. Sandrin, C. e Ugazio, U. L'inno "Andenken" di Hölderlin. Milano, Mursia, 1997); Hölderlins Hymne "Der Ister" (SS 1942). GA 53. Frankfurt an Main, Klostermann, 1984 (trad. it. Sandrin, C. e Ugazio, U. L'inno "Der Ister" di Hölderlin. Milano, Mursia, 2003).

5 Per un primo sguardo sulla relazione intessuta da Heidegger con la poetica hölderliniana si rimanda a ToSCANI, F. Poesia e pensiero nel "tempo di privazione". In cammino con Hölderlin e Heidegger. Pistoia, Petite Plaisance, 2010. Cfr. inoltre MoRETTI, G. Il poeta ferito. Hölderlin, Heidegger e la storia dell'essere. Imola, La Mandragora, 1999.

6 Non è casuale il fatto che Andenken sia anche il titolo di un inno composto da Hölderlin tra il 1803 e il 1805, che Heidegger interpreta e fa proprio in più circostanze, in particolare durante il corso di lezioni tenuto a Friburgo nel semestre invernale 1941/42 (Hölderlins Hymne "Andenken". Op. cit.). Si veda altresì il lungo testo intitolato Andenken scritto per la Tübinger Gedenkschrift in occasione del centenario della morte di Hölderlin (oggi in Erläuterungen zu Hölderlins Dichtung. Op. cit. pp. 79-151).

7 Heidegger, M. "Fenomenologia e teologia", in Segnavia. Trad. F. Volpi. Milano, Adelphi, 2008, p. 28. 
oggettivante, non è cioè qualcosa che si esaurisce o si consuma in rappresentazioni determinate.

Posso certo considerare la statua di Apollo nel museo di Olimpia come un oggetto di rappresentazione scientifico-naturalistica, posso misurare fisicamente il peso del marmo, così come posso esaminarlo nella sua composizione chimica. Ma questo pensare e questo parlare oggettivanti non vedono l'Apollo nella bellezza del suo mostrarsi e nel suo apparire in essa come immagine del dio ${ }^{8}$.

L'esempio sopra riportato consente al filosofo di misurarsi con quella che per lui è la piena natura del Denken. È vero -sottolinea Heidegger- che la statua di Apollo può essere pensata quale Objekt, come "oggetto" definito (stanziato in un luogo determinato e in un tempo preciso), ad esempio attraverso una lettura eminentemente scientifica che tiene in considerazione determinate caratteristiche fisico-chimiche come il peso, l'altezza o la composizione chimica. In questo senso, allora, l'opera d'arte in questione risulta essere Gegen-stand, ovverosia l'oggetto che fermamente sta di fronte agli occhi di un soggetto sondante e rappresentante. Ciononostante, è vero anche che, al di là di una interpretazione puramente empirica, siffatta statua conserva incalcolabili significati ed espressioni che hanno permesso ad essa di manifestarsi apertamente e venire all'essere. Pensare l'opera secondo questa prospettiva significa distaccarsi dalla sfera del pensiero rappresentativo; allontanarsi cioè dalla pretesa di calcolare, e in un certo senso controllare o manipolare, la cosa innanzi a sé. In ciò consiste lo Andenken, che nella riflessione heideggeriana si dà come l'autentico pensare che risponde all'esigenza di uscire da una certa visione del mondo (Weltanschauung) per cogliere l'ente in quanto tale:

Oggettivanti sono solo il pensiero e il linguaggio delle scienze naturali. Se ogni pensare fosse già di per sé oggettivante, allora sarebbero senza senso le forme delle opere d'arte, perché non potrebbero mai mostrarsi ad alcun uomo, dal momento che questi trasformerebbe subito in oggetto ciò che appare, e così impedirebbe all'opera d'arte di manifestarsi. L'affermazione che ogni pensiero in quanto pensiero è oggettivante, è senza fondamento. Essa si basa su una disattenzione per i fenomeni e tradisce una mancanza di coscienza critica9 . 


\section{Il poetare (Dichten) come forma eminente del pensare (Denken).}

Il primo vero grande confronto di Heidegger con l'opera poetica di Hölderlin -"un poeta strano, se non addirittura misterioso"10 - va tracciandosi nel corso friburghese del semestre invernale 1934/35 intitolato Hölderlins Hymnen "Germanien" und "Der Rhein", il cui intento generale è senz'altro quello di individuare, attraverso una singolare lettura del testo hölderliniano, la natura del linguaggio poetico. Per raggiungere siffatto risultato, il filosofo avanza preliminarmente un'osservazione critica nei confronti del vecchio assunto secondo cui la poesia, occupandosi di qualcosa che manifestamente non genera ricchezza materiale, sarebbe niente più che un ornamento estetico paragonabile al gioco emotivo "innocente" (unschuldig) o al "lieto svago" (fröhliche Zerstreuung) che offre all'uomo l'opportunità di mettere temporaneamente tra parentesi i problemi reali e concreti della quotidianità. Da questo punto di vista, l'insistente lettura di certi inni e lettere di Hölderlin risponde all'esigenza heideggeriana di ripensare radicalmente ciò che si chiama "poesia", la cui natura non risulta essere affatto ludica; cosicché

l'atteggiamento verso di essa non è la distensione giocosa che rende dimentichi di se stessi, bensì il risveglio e il cotrascinamento dell'essenziare più proprio del singolo, attraverso cui egli retrogiunge al fondamento del suo esserci ${ }^{11}$.

Lungi, allora, dall'essere un mero distacco emotivo dalla realtà, il discorso poetico ha in sé per Heidegger la peculiarità di penetrare nel cuore della realtà stessa, facendo emergere tutta la problematicità della natura umana. Ragion per cui esso è propriamente Erweckung e Zusammenriß, "risveglio" e "cotrascinamento" (nel senso del "riepilogare", del "mettere insieme", del "trascinare verso"), due voci per mezzo delle quali il filosofo mira ad esprimere l'originalità del Dichten, che consiste latamente nel rivelare ed indicare il carattere problematico dei fenomeni. In tal guisa, nell'opera poetica l'uomo consegue la possibilità di "tendere all'indietro" o "retrogiungere" (zurückreichen) verso le cose stesse in modo da coglierne l'aspetto autentico o originale. Per questa ragione, Heidegger non esita a designare il poeta come colui "che ci conduce all'origine o che la indica"12. Ogni discorso poetico è dunque un discorso che scava l'origine della cosa, ponendo in questione la relazione essenziale dell'uomo con essa.

Ora, promuovendo una riflessione sull'esperienza originaria del poetare, con il fine di sostenere una corrispondenza tra pensare e poetare, il filosofo recupera l'etimo del verbo

10 HeIdegger, M. "La poesia", in La poesia di Hölderlin. Op. cit. p. 219.

11 HeIdegger, M. Gli inni di Hölderlin "Germania" e "Il Reno". Op. cit. p. 8.

12 lb. p. 4. 
dichten, legandolo all'antico termine alto tedesco tihtôn, che a sua volta è connesso al latino dictare, cioè "dettare" nel senso dell'insistere su qualcosa, del fare in modo che qualcosa venga fissato per iscritto, dello "stendere linguisticamente qualcosa, sia esso un saggio, un resoconto, un trattato, una citazione o una petizione, una canzone o quel che si vuole"13. Perché emerga il carattere principiale del dictare, Heidegger pone segnatamente l'accento sul fatto che il verbo in questione altro non è che un intensivo di dicěre e, in quanto tale, va interpretato come Sagen, dire continuamente svelante-mostrante. Si tratta, insomma, di uno specifico dire che, svelando e mostrando ripetutamente altro, appare subordinato e soggetto a qualcosa che lo precede, ad un dire originario o primordiale: d'altronde, il dettato è in senso stretto ciò che scaturisce dall'ascolto attento di un dire anteriore.

Soffermandosi fondamentalmente sulla svelante azione del dictare, Heidegger prende così in considerazione il suo derivato greco dèiknymi che indica espressamente il "porre alla vista", il "rendere manifesto", il "mostrare". Da quanto scritto si ricava, allora, che il verbo dichten e il corrispondente sostantivo Dichtung risultano in fondo legati ad un atteggiamento che consiste nel manifestare e fare vedere qualcosa. Allo stesso tempo, siffatto atteggiamento appare eminentemente poietico e produttivo. Questo fa sì che la composizione poetica (in quanto Dichtung) venga in un certo modo pensata quale poíesis, dal verbo greco poieîn che significa "fare", "produrre" e, in un certo senso, "condurre all'essere" (eis ousían) ${ }^{14}$. Emerge così un discorso che interpreta il Dichten come un "produrre" nel senso del latino producere, vale a dire come Vollbringen, un "realizzare" o "portare a compimento" che bringt vor ("porta in luce") qualcosa dall'invisibile, lasciando che esso si mostri nella pienezza del proprio essere. Tutto ciò si dice in greco téchne:

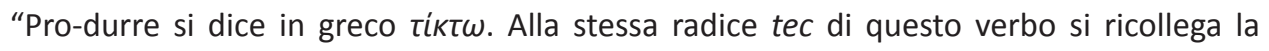
parola $\tau \varepsilon ́ \chi v \eta$, tecnica" ${ }^{15}$. Perciò, facendo riferimento a diversi passi dell'Etica Nicomachea aristotelica ${ }^{16}$, Heidegger parla della téchne come di ciò che concerne il "far venire

$13 \mathrm{lb}$. p. 33. Sulla radice etimologica del tedesco dichten si veda anche KLUGE, F. Etymologisches Wörterbuch. Berlin, De Gruyter, 1960, p. 178.

14 Heidegger riprende qui alcune considerazioni platoniche sul significato di "fare" in quanto poieîn. Nel Simposio (205b), infatti, Platone afferma che la poíesis è "ciò per cui qualcosa passa dal non essere all'essere" (he gár toi ek toû mè óntos eis tò òn iónti hotoioûn aitía pâsá esti poíesis). Con ciò si intende, dunque, l'attività produttiva che, in quanto tale, sta a fondamento del passaggio radicale da ciò che innanzitutto non è all'essere, dall'orizzonte dell'invisibile a quello del visibile. Per questa ragione, la poíesis è fondamentalmente pensata come presupposto di ogni attuazione e divenire. Al riguardo, si vedano anche le battute iniziali del Sofista (219b 4-7).

15 HeIdegger, M. "Costruire abitare pensare", in Saggi e discorsi. Trad. G. Vattimo. Milano, Mursia, 2015, p. 106.

16 ARISTOtele. Etica Nicomachea. 1140a 1-23; 1141a 9-12. 
all'esistenza" (ghenesis), il "far apparire qualcosa tra le cose presenti (in das Anwesende), come questo o quello, in questo o quel modo" ${ }^{17}$.

Questo lungo e tortuoso cammino filologico si inserisce pienamente nella riflessione circa il pensiero poetante (dichtendes Denken). Attraverso di esso, Heidegger ambisce di fatto a rileggere l'essenza (Wesen) della poesia in virtù delle sue diverse derivazioni da poíesis e da dictare. Di conseguenza, la poesia viene heideggerianamente pensata come il "pro-dotto" che, per opera di un "dire svelante" (dictare), ha la prerogativa di indicare la manifestatività di qualcosa, portando, conducendo (producendo) qualcosa alla vista, lasciando "apparire, indicandolo, l'invisibile"18. Sulla base di queste affermazioni, dunque, Heidegger chiarisce ancora una volta che il discorso poetico, e in generale quello artistico, "non è soltanto il costrutto linguistico sottomano e dotato di senso e bellezza"19; il che significa altresì affermare che esso "non è l'espressione linguistica di esperienze vissute psichiche"20. Piuttosto, quello poetico viene compreso quale wesentliche Sprache, il "linguaggio essenziale" che manifestamente apre all'innovazione ontologica e che per questo, lungi dall'essere uno Spiel virtuoso o sentimentale, si fa Stiftung: fondamento e "istituzione dell'essere" ${ }^{21}$.

In questo senso Heidegger legge l'ultimo verso dell'inno Andenken di Hölderlin che così recita in tedesco: Was bleibt aber, stiften die Dichter ${ }^{22}$. Nella celebre conferenza romana del $1936^{23}$ esso è interpretato come uno dei "detti-guida del poeta sulla poesia" 24 destinato "a poetare (dichten) espressamente l'essenza stessa della poesia"25. La ripresa di questo verso serve ad Heidegger per riflettere accuratamente sulla natura ontologica della poesia e sull'azione nominante-istituente dei poeti, i quali, attraverso il loro inneggiante canto (Gesang), pervengono a schiudere e "nominare" (nennen) le cose, generando una

17 HeIdegger, M. "Costruire abitare pensare", in Saggi e discorsi. Op. cit. p. 106.

18 HeIDEgGeR, M. "Terra e cielo di Hölderlin", in La poesia di Hölderlin. Op. cit. p. 194.

19 HeIdegger, M. Gli inni di Hölderlin "Germania" e "Il Reno". Op. cit. p. 33.

$20 \mathrm{lb}$.

$21 \mathrm{lb}$. p. 37.

22 "Ma ciò che resta, fondano i poeti" (HöLderlin, F. Poesie, ed. it. a cura di G. Vigolo. Milano, Mondadori, 1986, pp. 236-237). Si veda anche HÖLDERLIN, F. Tutte le liriche, ed. it. a cura di L. Reitani. Milano, Mondadori, 2001, pp. 344-345.

23 Si tratta della conferenza intitolata Hölderlin und das Wesen der Dichtung tenuta il 2 aprile del 1936 a Roma, a Villa Sciarra, nella sede dell'Istituto Italiano di Studi Germanici. Nel dicembre dello stesso anno, Heidegger ne fa pubblicare il testo nella rivista bavarese Das innere Reich, 3 (1936, pp. 1065-1078). Oggi lo scritto è raccolto nel volume Erläuterungen zu Hölderlins Dichtung. Op. cit. pp. $33-48$.

24 HeIdegGer, M. "Hölderlin e l'essenza della poesia", in La poesia di Hölderlin. Op. cit. p. 43. 25 Ib. p. 42. 
nuova visione di esse. Fondamentale è qui per il filosofo cogliere il significato precipuo del tedesco nennen, che rimanda dapprincipio all' “indicare” (Zeigen) nel senso dell'additare, del rendere noto qualcosa mostrandolo. La poesia assume così i contorni dell'arte della parola che si fa generatrice di una dimensione nella quale le cose vengono mostrate e colte nello splendore della loro pienezza e non rappresentate secondo una logica di tipo oggettuale o strumentale.

Sostenere, allora, che la poesia possieda il carattere della Stiftung (da stiften, "istituire") vuole dire per Heidegger perlomeno tre cose. E cioè che essa è da un lato istaurazione, dall'altro donazione e cura. L'azione del poeta, infatti, è sempre al tempo stesso creante, donante e curante. Egli istituisce (svela) una nuova realtà singolare, custodendola e salvaguardandola con la sua parola poetica, offrendo all'uomo la straordinaria opportunità di abitarla. II poetare del poeta consiste dunque nel nominare ed istituire l'apertura che di volta in volta prepara un abitare, disponendo (nel senso dello einräumen) e liberando (nel senso dello freigeben) spazi di senso in grado di interrogare la natura dell'uomo, mostrando tacitamente il fondamento di detta natura. Così, in definitiva, il poetare in quanto Dichten e Urdichten ("poetare originario") viene ad essere compreso essenzialmente da Heidegger come la forma eminente del pensare.

\section{La Kunstfrage. La natura del discorso artistico.}

Per uscire dalle consuetudini del pensiero metafisico e dell'estetica tradizionale, Heidegger sviluppa una riformulazione del problema della poesia e dell'arte in generale attraverso una riflessione circa la valenza ontologica dell'opera d'arte, che ritroviamo in particolare in Der Ursprung des Kunstwerkes ${ }^{26}$. Qui, nel tentativo di cogliere l'“origine” (Ursprung) dell'opera artistica come il dove "dal quale e attraverso il quale una cosa è, nel proprio modo, ciò che è" ${ }^{27}$, il filosofo pone immediatamente l'accento sulla relazione tra Künstler e Werk. A partire da questa sottile relazione, egli delinea la sua indagine estetica -se per "estetica" si intende segnatamente l'esame che tiene conto del nesso tra la riflessione artistica e l'ontologia, tra téchne e alétheia- preoccupandosi di riconsiderare i termini "artista" e

26 Oggi in "Der Ursprung des Kunstwerkes". Holzwege, GA 5. Frankfurt am Main, Klostermann, 1977, pp. 1-74. La versione italiana a cui qui si fa riferimento è: L'origine dell'opera d'arte. Trad. I. De Gennaro e G. Zaccaria. Milano, Marinotti, 2012.

Per una ricerca impegnata a cogliere la portata della questione dell'arte nel pensiero di Heidegger si veda esemplarmente KoCKELMANS, J. J. Heidegger on art and art works. Dordrecht, Nijhoff, 1985. Si vedano altresì Young, J. Heidegger's Philosophy of Art. Cambridge, Cambridge University Press, 2001; MARAFIOTI, R. M. La questione dell'arte in Heidegger. Soveria Mannelli (CZ), Rubbettino, 2008; BALAZUt J. "La thèse de Heidegger sur l'art", en Nouvelle revue d'esthétique 5, 2010, pp. 141-152.

27 HEIDEGGER, M. L'origine dell'opera d'arte. Op. cit. p. 3. 
"opera" come parti insolubili di un circolo ricomprendente (Kreisgang) in cui nessuno dei due è senza l'altro ed in cui entrambi, di conseguenza, si rinviano vicendevolmente.

Attraverso una personale lettura del dipinto di van Gogh raffigurante un paio di scarpe, Heidegger si inoltra nella domanda sull'arte per cercare di cogliere la cifra del discorso artistico. II quadro del pittore diventa un pretesto per potere dare luogo ad una interpretazione che si spinga ad esaminare ciò che "accade" (geschieht) all'interno dell'opera in questione e, latamente, in ogni opera d'arte: "Che cosa accade qui? Che cos'è all'opera nell'opera?"28. Sottraendo il mezzo, cioè le scarpe, dal contesto abituale, l'opera oltrepassa l'orizzonte della quotidianità, aprendo e lasciando vedere un mondo ricco di significati e relazioni singolari. Nella vita di tutti i giorni le scarpe in quanto Zeug, "strumento" o "attrezzo", servono alla contadina per lavorare il campo ${ }^{29}$. Esse hanno evidentemente un fine pratico. La contadina tutti i giorni le indossa senza però riflettere sul loro valore d'uso. Al contrario, ella si fida dell'attrezzo che impiega e consuma quotidianamente. In virtù di questo particolare stato, che Heidegger chiama die Verlässlichkeit ("la fidatezza"), la contadina si pone continuamente in relazione ad un mondo specifico al quale lei pienamente aderisce e sa, in fondo, di appartenere:

Ogni volta che, a tarda sera, presa da una stanchezza dura ma sana, la contadina ripone le scarpe, per riprenderle già quasi subito nell'ancora buio crepuscolo del mattino, o anche quando, in un giorno di festa, vi passa solo accanto -ogni volta lei sa tutto questo senza avere nulla da osservare o considerare ${ }^{30}$.

L'opera d'arte, invece, estrapola l'oggetto dal suo contesto, mostrandone la natura, rilevando il fatto che l'oggetto impiegato quotidianamente si inserisce all'interno di un mondo di senso e connessioni. Qui si sviluppa una riflessione profonda sulla natura della cosa, al di là del suo valore pratico, che permette di lasciare vedere la strumentabilità delle cose che circondano il quotidiano. Rivelando il carattere essenziale dell'ente in quanto tale, l'opera non fornisce una precisa spiegazione dell'ente o del mezzo, bensì fa sì che das Zeugsein des Zeuges ("I'attrezzità", "I'esser-attrezzo dell'attrezzo") si mostri per ciò che è, aprendo e facendo insorgere l'ente in quanto ente.

\section{Ib. p. 43.}

29 Affermando l'appartenenza del paio di scarpe ad una contadina (Bäuerin), Heidegger abbozza una libera interpretazione del quadro che è stata, a ragione, oggetto di critiche molto severe da parte di numerosi storici dell'arte (tra tutti Meyer Schapiro). Cionondimeno, essa ha comunque contribuito ad alimentare un vivace dibattito ripreso nella seconda metà del Novecento da intellettuali come Lacan, Derrida, Jameson, Cacciari, Boehm. Per questo aspetto cfr. ad esempio PanATtonI, R.; Grazıoli, E. (a cura di). Le scarpe di Van Gogh. Milano, Marcos y Marcos, 2013.

30 HEIDEGGER, M. L'origine dell'opera d'arte. Op. cit. p. 39. 
Da quanto finora detto, è possibile ricavare già sufficienti elementi per svolgere alcune considerazioni intorno alla portata ontologica del discorso artistico secondo Heidegger. Da un lato, giacché si misura con la natura dell'ente, l'arte non può ridursi affatto a semplice mìmesis, cioè a imitazione o riproduzione di qualcosa di già esistente. Allo stesso tempo, emancipandosi sempre dal rapporto con il suo autore, il significato essenziale del discorso artistico non ha nulla a che vedere con lo stato emotivo del singolo o con il suo talento. In base a queste considerazioni, dunque, il paio di scarpe dipinto da van Gogh, rompendo con l'ordinaria rappresentazione della vita, dello spazio e del tempo quotidiani, non si limita a riprodurre allegoricamente un oggetto, bensì porta all'essere qualcosa di nuovo, un nuovo orizzonte di senso che contribuisce a rivelare e "stanziare un mondo" (eine Welt aufstellen). Se ne ricava che l'ontologia dell'arte consiste nell'istituire ogni volta la realtà e non riprodurla o imitarla. Si tratta, più precisamente, di un istituire pensato secondo un triplice senso, e cioè come Schenkung ("donazione"), Gründung ("fondazione") e Anfang (“inizio"). Questi tre aspetti della Stiftung stanno alla base dell'essenza dell'arte quale istituzione del mondo nell'opera. Le stesse scarpe, usurate e sporche di fango, si mostrano nel celebre dipinto quali produttrici e rivelatrici del mondo contadino e della sua verità.

Uno dei due tratti specifici dell'opera d'arte è dunque l'erezione di un mondo che, però, non è interpretato come una realtà definita che alberga "stabilmente di fronte a noi, qualcosa che possiamo incontrare come un oggetto, o fissare come un obiettivo" ${ }^{13}$; la nozione di Welt, piuttosto, è qui pensata come l'insieme dinamico delle decisioni che concernono e coinvolgono l'esistenza storica di una comunità:

Là dove vengano prese le decisioni costitutive della nostra istorialità dispensale, decisioni da noi assunte o abbandonate, misconosciute o nuovamente esperite nell'interrogare, proprio là il mondo si fa mondo ${ }^{32}$.

Il secondo carattere precipuo dell'opera d'arte riguarda l'affermazione della terra. II verbo utilizzato da Heidegger per indicare questo "affermare" è stellen. Esso è qui pensato nel senso del latino ad-firmare, che possiamo tradurre con "posare", "lasciare giacere dinanzi". L'arte, allora, stellt la terra, lascia cioè che essa insorga ed emerga in tutta la sua pienezza, senza eroderla o consumarla, ma affermandola e custodendola all'interno di un mondo. In altre parole: "l'opera lascia che la terra sia una terra" ${ }^{33}$. Al riguardo, l'esempio del tempio greco permette ad Heidegger di esplicitare meglio la sua posizione estetica. Nel mezzo di una valle dirupata, il tempio si erge e dà origine, aprendolo, ad un

31 lb. p. 61.

32 lb. p. 63.

$33 \mathrm{lb}$. p. 65. 
mondo pregno di valori in cui va sviluppandosi gradualmente la storia della comunità degli uomini; al contempo, la struttura architettonica esalta i materiali che la compongono -la pietra, il colore, ecc.-, dando loro una forma più propria, rivelandoli e lasciandoli apparire nella loro essenza, inserendoli e mantenendoli perciò "nell'estensione (in das Offene) di un mondo" 34 .

Mondo e terra, Welt e Erde, appaiono dunque nell'opera d'arte in ogni modo inseparabili. Essi si rivelano insieme come due poli "costitutivamente di-vergenti, eppure mai separati" 35 . Se il primo termine costituisce infatti l'apertura nella quale una comunità storica di uomini è inserita e compresa, il secondo, lungi dall'indicare "una massa materiale sedimentata" ${ }^{36}$ o una "nozione puramente astronomica" ${ }^{37}$, costituisce invece lo sfondo oscuro e celato -das wesenhaft Sichverschließende ("ciò che essenzialmente si chiude da sé")-, fonte e sorgente insondabile di ogni apparire, che continuamente sfugge a qualsiasi tipo di logica categorizzante in quanto autenticamente non è alcunché di costruito dall'uomo. Questi due termini stanno nell'opera in relazione. Si tratta di una relazione esclusiva che è Streit, ovverosia una "controversia" o "contesa" (pólemos) in cui i due contendenti non si annullano affatto, ma si richiamano a vicenda, congiungendosi e in pari tempo distinguendosi. Per questa ragione, Heidegger afferma quanto segue: "II mondo si fonda sulla terra, mentre si erge terra attraverso un mondo"38. Il che significa da un lato che il mondo si apre e si invera su uno sfondo terreno, per cui "non può volare via dalla terra" ${ }^{39}$; dall'altro, la terra ha bisogno del mondo per manifestarsi compiutamente. Se è vero, pertanto, che il mondo weltet ("si fa mondo", si schiude in quanto tale) sulla terra, inscrivendo quest'ultima nella propria apertura, è vero anche che la terra, ergendosi attraverso il mondo, lo tiene in sé e lo avvolge nel proprio nascondimento ricoprente. Da questo punto di vista, ogni opera d'arte è interpretabile precisamente come eine Anstiftung dieses Streits, "un'istigazione della contesa" ${ }^{40}$. Detta Anstiftung è colta da Heidegger, sulla base della sua etimologia (dal verbo anstiften, derivato del latino instigare e del greco stizein), come una "provocazione" o uno "stimolo". Per cui risulta ancora più chiaro che la tensione tra mondo autoaprentesi e terra velante-custodente non è una contesa a partire dalla quale i due estremi concentrano tutte le proprie forze ed energie al fine di

$34 \mathrm{lb}$.

35 Ib. p. 71.

36 lb. p. 57.

$37 \mathrm{lb}$.

38 Ib. p. 71.

$39 \mathrm{lb}$. p. 73.

$40 \mathrm{lb}$. 
distruggere o annullare l'altro. Heidegger afferma così che Streit non è Riß; non è una "frattura" o uno "strappo" che lacera e separa una volta per tutte gli oppositori, bensì è ciò che massimamente raccoglie, congiunge ed esalta:

Fin troppo facilmente falsiamo l'essenza di quest'ultima (della contesa), confondendola con la discordia e l'ostilità, e determinandola, perciò, solo come sconvolgimento e distruzione. Nella contesa costitutiva, invece, i contendenti si esaltano via via reciprocamente ${ }^{41}$.

In questa tensione-provocazione esaltante si mette in moto incessantemente la verità; quest'ultima, secondo un'espressione cara al linguaggio heideggeriano, "è al lavoro" (ins Werk gesetzt), cioè "accade" (geschieht) e, accadendo nell'opera d'arte, dà origine a quell'“urto" (Stoß) che determina, ogni volta in modo assai singolare, una verità storica, una Geschichte. Nell’opera si manifesta la contesa come pólemos tra mondo e terra. Si tratta di una contesa avente carattere poietico che dà ritmo all'opera e conduce verso quella "fissazione" o "determinazione" (Feststellen) della verità, che consiste nel lasciare che la cosa si riveli per ciò che è. Questa "fissazione" non significa però un irrigidimento, un'immobilizzazione della verità nell'opera creata; al contrario, in un significato radicale, l'opera, e dunque la verità che essa disvela, non è mai rigida o statica, ma conserva in sé le tracce più sottili della tensione costante tra il mondo e la terra, attraendo e lasciandosi interrogare senza mai farsi oggettivare.

La Kunstfrage heideggeriana si inserisce all'interno di un più vasto progetto interessato a porre la domanda sulla verità. Secondo questa prospettiva, l'essenza dell'arte chiama in causa l'essenza della verità. Le due questioni, invero, appaiono intimamente correlate. Come già rilevato, ciò che è in opera nell'opera d'arte non è "una verità dell'esperienza pratica della vita o di un calcolo economico" ${ }^{42}$. Del resto, nella sua accezione essenziale la verità non è colta da Heidegger come "un carattere del conoscere e dell'asserire umani, e tanto meno non è un mero valore o un' 'idea' alla cui realizzazione -non si sa bene perchél'uomo deve aspirare"43. Addentrandosi nello studio della parola Wahrheit (dal verbo wahren, che indica un "custodire", un "tutelare" o un "salvare" nel senso del "perdurare" e del "mantenere a lungo"), il filosofo riscopre l'essenza della verità, pensandola nei termini della nozione greca alétheia, che a sua volta rimanda alla singolare dinamica in cui l'essere stesso delle cose appare e si rivela nella relazione intrinseca tra velamento e svelamento,

$41 \mathrm{lb}$. p. 71.

42 Heidegger, M. "Dell'essenza della verità", in Segnavia. Op. cit. p. 133.

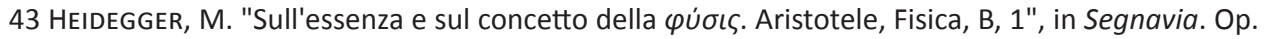
cit. p. 255. 
nascondimento e scoprimento. L'eccezionalità di questa relazione ogni volta si produce e si invera nell'opera. Cosicché la ricerca heideggeriana, scavando nella profondità del discorso artistico, permette di elaborare una nuova idea di arte.

A fondamento dell'elaborazione sta l'argomento secondo cui l'arte è la maniera privilegiata di parlare della verità. Di conseguenza, quello artistico è per Heidegger un linguaggio primariamente veritativo nel senso che ha sempre a che fare con la natura o con la verità dell'essente. L'arte, difatti, disvela la verità, facendo in modo che essa appaia e si trasmetta nella storia. Da questo punto di vista, Heidegger affronta con radicalità uno dei nuclei problematici dell'estetica tradizionale, avanzando la tesi secondo cui l'opera d'arte risulta come il luogo nel quale prende ogni volta forma "un avvenire, ossia un accadere, della verità" ${ }^{44}$. Ciò che propriamente nell'opera accade è das Geschehnis der Wahrheit ("I'accadere, l'evento della verità"):

L'arte è allora: il creante serbante inveramento della verità nell'opera (die schaffende Bewahrung der Wahrheit im Werk). L'arte è, quindi, un divenire (ein Werden) e un accadere (Geschehen) della verità ${ }^{45}$.

Qui, infatti, attraverso un linguaggio artistico, l'ente viene detto nella sua totalità senza essere ridotto a oggetto. Pertanto, facendo insorgere l'essere dell'ente, l'arte mostra apertamente l'in sé del fenomeno, ciò che esso è in verità, senza pretendere di descriverlo oggettivamente, come invece si impegna a fare, nelle sue diverse articolazioni, il linguaggio tecnico-scientifico:

L'attrezzità è stata trovata. Ma in che modo? Non attraverso la descrizione e la spiegazione di un paio di scarpe che giaccia effettivamente dinanzi; non attraverso un resoconto del loro processo di fabbricazione; e neppure osservando un loro concreto impiego, bensì unicamente recandoci dinanzi al dipinto di van Gogh. Il quadro ha parlato. Vicino all'opera, ci siamo di colpo ritrovati in un altro luogo rispetto a quello in cui siamo abituati a stare $^{46}$.

Cercando di cogliere l'essenza dell'arte (das Wesen der Kunst) nella capacità di rendere palese la verità dell'ente-cosa (Ding), facendolo emergere attraverso immagini, suoni o parole, Heidegger reinterpreta il problema di una fondazione ontologica dell'arte prendendo in considerazione il significato autentico di Dichtung. Nell'ottica

44 HEIDEgGER, M. L'origine dell'opera d'arte. Op. cit. p. 43.

45 lb. p. 119.

46 lb. pp. 41-43. 
della Kunstfrage, sviluppando un'analisi che procede oltre la quotidianità concreta dell'esistenza, Heidegger volge lo sguardo verso quel particolare linguaggio artistico grazie al quale le cose si manifestano per ciò che sono. In questo modo, egli tenta di superare i limiti dell'analitica esistenziale e di Sein und Zeit, ripensando la Seinsfrage non più a partire dalla centralità dell'esserci, bensì attraverso un linguaggio che nella sua radice ontologica viene ad essere recepito come Ereignis, "apertura originaria", "evento".

Arte in quanto Dichtung è perciò l'originaria creatività poetica (poíesis) che be-dingt la cosa, cioè dà voce ad essa, rendendola ciò che è. Così, la riflessione sullo stato dell'arte permette al filosofo, chiaramente influenzato dalla poesia di Hölderlin, di confrontarsi allo stesso tempo, in una maniera del tutto originale, con la questione circa la natura della cosa e il suo significato essenziale, mettendo in evidenza la portata fenomenologica di Ding e dei termini ad esso connessi (ad esempio das Dinghafte o das Dingsein). Seinsfrage, Kunstfrage e Dingsfrage si inseriscono dunque all'interno di una problematica in sé unitaria interessata a scavare in profondità nel senso delle cose e dei fenomeni, ponendo in luce tutta la problematicità dell'esistenza e la sua apertura.

Per tutto ciò, l'arte viene pensata da Heidegger come l'essenza del poetare che genera relazioni e verità profonde: "L'arte, in quanto è la messa in opera della verità, è dettatura (cioè Dichtung)" ${ }^{47}$. Essa, in quanto permette il manifestarsi dell'essere nella Gestalt, si misura costantemente con un invisibile, con un non detto, lasciando che esso emerga poco a poco come "pro-dotto". In questo senso, l'arte in quanto Dichtung si fa rivelatrice e insieme custode di una particolare verità, preservando la domanda originaria sul senso dell'essere, obliata dalla tradizione metafisica. Qui si delinea uno degli aspetti decisivi dell'estetica heideggeriana: I'unità di stiften e hüten ("istituire" e "custodire"), creazione e protezione, composizione e salvaguardia.

Attraverso il suo poetare, l'artista dichtet nella misura in cui rende visibile l'invisibile, compiendo la manifestazione dell'essere, annunciandolo e custodendolo nell'opera. Da questa prospettiva si capisce meglio l'intenzione heideggeriana di sottrarsi al rigore logico-scientifico per affermare una posizione filosofica che si faccia carico della Seinsvergessenheit perpetrata dalla metafisica e che, perciò, rimetta decisamente in discussione la questione dell'essere. Trattasi, de facto, di una posizione nella quale all'atteggiamento catturante e calcolante viene contrapposto un pensiero capace di recuperare il legame originario con l'essere e, allo stesso tempo, di cogliere il carattere evenemenziale della cosa, esprimendone la natura, la sua essenza.

47 Ib. p. 125. 
Così l'artista, in quanto Dichter, risulta ein Zeichen, cioè un "segno" o un "indice" che zeigt la verità dell'ente ${ }^{48}$. Egli si dà nella ricerca estetica heideggeriana come l'autentico pensatore (authentische Denker) che, avvalendosi di un linguaggio esclusivo, testimonia il "riferimento" (Bezug) dell'essere all'uomo. Nelle pagine del Brief über den Humanismus ${ }^{49}$, lo scritto indirizzato a Jean Beaufret nel dicembre del 1946, Heidegger prende seriamente in considerazione la domanda sul significato del Denken, centrale in tutto il suo Denkweg, sviluppando l'idea secondo la quale pensare vuole dire portare a compimento (nel senso del già visto Vollbringen) “il riferimento (Bezug) dell'essere all'essenza dell'uomo"50. Con questa affermazione, il filosofo tedesco intende cogliere la specificità del pensare nella capacità di "dispiegare qualcosa nella pienezza della sua essenza, condurre-fuori a questa pienezza, producere" ${ }^{\prime 51}$.

Detto in altro modo: pensare non significa produrre tecnicamente qualcosa, ad esempio oggetti o rappresentazioni mentali (a risuonare è, chiaramente, la critica al pensiero metafisico). L'essenza del pensare consiste, invece, nel realizzare, nell'istituire, nel portare alla luce e al linguaggio la verità dell'essere tramite un pensiero che è eminentemente dichtendes Denken. È nel linguaggio, dunque, che l'essere in senso proprio geschieht. Se ne ricava che la questione del linguaggio non va affatto separata dalla questione dell'essere. Ciò permette di descrivere l'evento linguistico in chiave ontologica come fenomeno problematico che "parla in quanto dice, cioè mostra" 52 . In tale prospettiva Heidegger parla della Sprache come della "casa dell'essere"53, capace di accoglierlo pienamente e testimoniarlo di volta in volta. Questo "testimoniare" (bezeugen) dice l'essere in un duplice modo: da un lato lo lascia apparire, conducendolo alla vista; dall'altro lo vela in sé, custodendone e difendendone l'integrità. Così, nell'inarrestabile

48 L'interpretazione heideggeriana dell'uomo (e in particolare dell'artista) come Zeichen è fortemente legata ai seguenti versi di Hölderlin tratti dall'inno Mnemosyne: "Ein Zeichen sind wir, deutungslos / Schmerzlos sind wir und haben fast / Die Sprache in der Fremde verloren"; in italiano: "Noi siamo un segno non significante, / indolore, quasi abbiamo perduto / nell'esilio il linguaggio" (HöLDERLIN, F. "Mnemosyne", in Le liriche, ed. it. a cura di E. Mandruzzato. Milano, Adelphi, 1977, p. 695). Per questo aspetto cfr. HeIDEg GeR, M. Che cosa significa pensare? Trad. U. Ugazio e G. Vattimo. Milano, Sugarco, 1988, pp. 43-46. Cfr. anche HEIDEgGER, M. "Rammemorazione", in La poesia di Hölderlin. Op. cit. p. 126.

49 Oggi in Heidegger, M. "Briefe über den Humanismus", in Wegmarken (1919-1961), GA 9. Frankfurt am Main, Klostermann, 1976, pp. 313-364 (trad. it. Volpi, F. Lettera sull'"umanismo". Milano, Adelphi, 2008).

50 HeIDEGGER, M. Lettera sull'"umanismo". Op. cit. p. 31.

$51 \mathrm{lb}$.

52 Heidegger, M. "Il cammino verso il linguaggio", in In cammino verso il linguaggio. Trad. A. Caracciolo. Milano, Mursia, 2014, p. 200.

53 HEIDEGGER, M. Lettera sull'"umanismo". Op. cit. p. 96. 
dialettica tra svelamento e velamento, rivelazione e nascondimento, si afferma e si realizza sempre il darsi dell'essere come Ereignis.

È nell'opera d'arte, è nel linguaggio artistico che tutto ciò va palesandosi segnatamente. L'opera stessa, infatti, attesta la presenza dell'essere, svelandolo e insieme velandolo. È qui che secondo Heidegger si rivela uno dei significati propri del pensare come Dichten, che riguarda il fare emergere e salvaguardare l'ente-cosa (e non sfruttarlo o esaurirlo). Ragion per cui l'artista, ovvero l'autentico pensatore, viene compreso più di ogni altro come "pastore dell'essere" ${ }^{54}$, istitutore e custode del suo significato più essenziale.

\section{L'evento del nichilismo e la necessità di riallacciare il dialogo interrotto con l'essere.}

La riflessione sulla Dichtung consente ad Heidegger di avanzare una proposta filosofica che si misura con le problematiche e le necessità della contemporaneità, mettendo in evidenza la povertà e lo smarrimento dell'uomo nell'età della tecnica. Questa condizione è espressa dal filosofo con il termine Heimatlosigkeit, attraverso cui egli arriva a segnalare una mancanza, una perdita nella relazione tra l'uomo e l'essere ${ }^{55}$. Qui, la parola Heimat è compresa in un senso del tutto singolare e risulta perciò difficilmente traducibile in altre lingue. Essa viene generalmente tradotta in italiano con "casa" o "patria". Si tratta, più sottilmente, della "casa" intesa non nel senso del mero edificio, ma della dimora, nella quale sono raccolti e custoditi i nostri più profondi ricordi ed affetti, che ha a che fare con le nostre origini. Da questo punto di vista, la categoria di Heimatlosigkeit è pensata da Heidegger come sintomo della tecnica moderna e conseguenza drammatica della deprivazione di uno spazio abitativo originario ${ }^{56}$; una perdita che si traduce altresì nell'incapacità da parte dell'essere umano di comprendere l'interrogazione circa la peculiarità della propria natura e della propria posizione nel mondo.

L'indagine sul discorso artistico e la necessità di ripensare il significato del Denken celano in sé, dunque, tutta la critica al pensiero tecnico-scientifico come compimento e

\section{4 lb. p. 56.}

55 Lo sviluppo del concetto di Heimatlosigkeit (comunemente tradotto con "spaesatezza") -insieme alle altre due categorie heideggeriane di Ratlosigkeit ("disorientamento") e Bodenlosigkeit ("sradicamento")- rappresenta uno dei motivi ricorrenti nella speculazione matura di Heidegger. Cfr. ad esempio HeIDEGgeR, M. Lettera sull'"umanismo". Op. cit. Sulla questione si veda VolPI, F. "'Noi senza patria': Heidegger e la Heimatlosigkeit dell'uomo moderno", en Revista Portuguesa de Filosofia 59, 2003, pp. 1261-1267.

56 Sulla base di questa interpretazione, Derrida descrive la categoria heideggeriana di Heimatlosigkeit come absence du chez-soi, "la mancanza-di-casa-propria" (DERRIDA, J. Psyché. Invenzioni dell'altro. Vol. 2. Trad. R. Balzarotti. Milano, Jaka Book, 2009, p. 112). 
culmine della metafisica occidentale. Si tratta, cioè, di una critica a quel sistema di idee e valori che, facendo di qualunque essente un oggetto da provocare, cioè un "fondo" (Bestand) da impiegare (bestellen) e consumare (verbrauchen), sviluppa un'indagine sul reale la cui conseguenza drastica è il graduale e radicale abbandono dell'essere, il fatto evidente che dell'essere non ne è più nulla. Secondo questa prospettiva, Heidegger coglie nello spirito della metafisica il manifestarsi di un fenomeno, esso stesso nichilistico, che si esplica compiutamente nell'idea nietzschiana di "volontà di potenza" (Wille zur Macht), attraverso la quale viene posto l'accento sulla pretesa da parte della soggettività umana di cogliere e dominare la totalità del reale (e dunque di imporre la propria forza alle cose), e nelle logiche produttive ed efficienti del mondo della tecnica.

Per comprendere fino in fondo la radice della metafisica, Heidegger ritiene imprescindibile confrontarsi con la questione del nichilismo ${ }^{57}$ per ripensare con radicalità le lacerazioni e le profonde inquietudini dell'uomo nell'età della tecnica ${ }^{58}$. Attraverso

57 II problema del nichilismo viene affrontato da Heidegger in modo approfondito soprattutto nei corsi di lezione dedicati a Nietzsche. Cfr. in particolare HeIDEgGER, M. Nietzsche 1 (1936-39). GA 6.1, Frankfurt am Main, Klostermann 1996 e Nietzsche 2 (1939-1946). GA 6.2. Frankfurt am Main, Klostermann 1997 (Nietzsche. Trad. it. Volpi, F. Milano, Adelphi, 1994).

Nel confronto con il pensiero nietzschiano, Heidegger sviluppa una profonda riflessione filosofica inerente la questione del nichilismo, fornendone una originale interpretazione, ricomprendendola come una delle categorie privilegiate per interpretare il destino dell'Occidente. Al riguardo, cfr. VolPI, F. I/ nichilismo. Bari, Laterza, 2009. pp. 83-107. Sulla questione del nichilismo sviluppata da Heidegger nel confronto con Nietzsche si veda esemplarmente VITIELLO, V. Utopia del Nichilismo. Tra Nietzsche e Heidegger. Napoli, Guida, 1983.

58 Precarietà, incertezza, squilibrio, spaesamento, mancanza di riferimenti, disorientamento sono tutti stati legati direttamente al concetto di nichilismo. Esso è senza dubbio un fenomeno che incide in maniera tagliente nel cammino dell'uomo contemporaneo.

Anche se il termine "nichilismo" viene in un certo modo utilizzato già verso la fine del Settecento (in particolare da Jacobi, Schlegel, Jean Paul, Schelling, Hegel), esso, tuttavia, comincia seriamente ad affermarsi a partire dalla prima metà del XIX secolo nell'ambiente culturale russo. Nel 1829, il critico letterario Nadeždin scrive un articolo intitolato L'adunata dei nichilisti (nigilistov). Scene della fiera letteraria, utilizzando per la prima volta la parola "nichilismo" per descrivere quella categoria di uomini che non sa e non capisce nulla. All'incirca dieci anni più tardi un altro intellettuale russo, Michail Nikiforovič Katkov, si scaglia polemicamente contro i collaboratori della rivista Sovremennik, accusandoli direttamente di nichilismo, cioè di non credere a nulla e di essere indifferenti a qualsiasi valore e/o principio. Nel 1862, lo scrittore Ivan Turgenev scrive il romanzo Padri e figli, nel quale viene narrata la lotta tra la vecchia e la nuova generazione, tra i padri attaccati agli antichi valori e i figli desiderosi di opprimere il vetusto sistema tradizionale. In questa contesa tra due differenti visioni del mondo, emerge la figura brillante di Bazarov, giovane medico di provincia che si ribella contro quella nobiltà russa incapace di comprendere le reali esigenze della società del tempo. Egli viene ad essere definito da Turgenev come il nichilista che nega le categorie del passato, I'antisistema che, negando e distruggendo, drammaticamente avverte su di sé il peso della creazione di una nuova e più lucente realtà. Con Turgenev, dunque, l'idea del nichilismo si propaga e comincia ad essere appresa (recepita) in quanto materia di discussione pubblica. Tanto è vero che nella Russia del secondo Ottocento il nichilismo diviene un fenomeno incandescente capace di infiammare 
un'analisi del tutto originale, il filosofo coglie l'essenza del nichilismo non tanto come un avvenimento contingente rappresentabile in termini storiografici, bensì come l'evento inerente la storia dell'essere (Geschichte des Seins). In conseguenza di ciò, la storia della metafisica viene ad essere identificata come storia del nichilismo entro la quale va articolandosi e realizzandosi l'evento dello svuotamento dell'essere, i cui innegabili effetti sono la riduzione delle cose a oggetti e lo sfruttamento dei viventi, e tra i viventi gli uomini, a risorse dominabili e utilizzabili.

Detto in altro modo, con il concetto di nichilismo la riflessione heideggeriana intende porre l'accento su ciò che via via si è imposto come condizione normale della nostra esistenza, finendo per scandire pienamente i ritmi e le regolarità della società contemporanea. Così, lo sviluppo della metafisica in quanto evento nichilistico -da Socrate e Platone in avanti, passando per la Scolastica, il cartesianesimo e la scienza moderna, fino a toccare le dottrine psicoanalitiche e il platonismo rovesciato di Nietzsche- ha gradualmente ma inesorabilmente trasformato la relazione dell'uomo col mondo esterno, generando un sistema imperniato sull'interpretazione dell'essere come idea e strumento nelle mani del soggetto umano. Ma è soprattutto nell'epoca del dominio planetario della tecnica, "cioè dell'incondizionato premere dell'essente verso la consumazione dell'usura" ${ }^{59}$, che Heidegger coglie la radice del nichilismo come ciò che arreca la chiusura e l'estraniamento nei confronti dell'essere.

È questa l'epoca in cui "il mondo è divenuto non-mondo"60. L'idea del "non-mondo" rappresenta per il filosofo una metafora attraverso la quale riflettere su una negazione, un abbandono, che si traduce letteralmente in un fare a meno dell'essere (del suo significato

la società e la politica nazionale, finendo per designare un movimento di ribellione sociale che si oppone al potere autoritario zarista e ne propone l'abbattimento.

Innegabilmente, però, è con Dostoevskij che il nichilismo affiora prepotentemente come problema ideologico e sociale. Nei suoi romanzi, il dilemma nichilistico lascia al lettore un gusto amaro. Il disfacimento dei vecchi valori è avvertito in tutta la sua inquietezza e criticità. I personaggi di Dostoevskij si mostrano ribelli e insofferenti ai fondamenti tradizionali della società russa. Essi sono gli autentici nichilisti che ogni volta mettono in discussione il sistema, sfidandolo ed attaccando Dio, che del sistema è il punto di riferimento, fino a negarlo. Una ribellione e una profonda insofferenza che sottilmente ritroviamo tra le pagine nietzschiane, nelle quali l'evento disorientante del nichilismo va affermandosi gradualmente come uno dei temi urgenti e principali per la riflessione filosofica.

Per un'analisi storico-concettuale impegnata a riflettere sulle radici del fenomeno del nichilismo cfr. nello specifico VolPI, F. II nichilismo. Op. cit. Sulla questione, si veda l'originale e ormai celebre studio condotto da Severino sull'affermazione del nichilismo come storia dell'identificazione dell'essere con il nulla (SEverino, E. L'essenza del nichilismo. Milano, Adelphi, 1982). Cfr. altresì SonCINI, U. "La tematica del nichilismo in Nietzsche, Heidegger, Severino", in Divus Thomas 102, 1999, pp. 182-223. 59 HeidegGer, M. "Oltrepassamento della metafisica", in Saggi e discorsi. Op. cit. p. 60.

$60 \mathrm{lb}$. 
originario) nel nome della costante produzione tecnica. Quest'ultima risponde a sua volta al paradigma della Wirkung (dell' "efficacia"), alla cui base stanno i complessi procedimenti di consumazione ed organizzazione. Nella dialettica circolare tra ente consumato ed ente organizzato va estendendosi il "non-mondo" come lo spazio in cui il dialogo con l'essere è interrotto, non è più in vigore. Per questo motivo il filosofo afferma che, trasformato e ridotto sempre in qualcos'altro, "I'essere è bensì presente (west), ma senza un proprio vigere (Walten)" ${ }^{61}$. Ragion per cui, svuotato del suo più autentico significato, nell'età della tecnica "I'essere si lascia andare nel fare e nel macchinare"62. Esso viene compreso, quindi, nella forma dell'ente organizzato e consumato. Si potrebbe dire, in altri termini, che l'essere, seppur ridotto ad altro, rimane pur sempre presente, ma come ente o come oggetto funzionale alla produzione. Conseguenza diretta di questa riduzione è la rivelazione di uno stato di Heimatlosigkeit, della spaesatezza come radicale assenza dell'essere e incapacità di pensarlo nuovamente -al di là dell'aspetto meramente produttivo della funzionalità tecnica- nella sua sacralità e inviolabilità come Unberechenbaren, l'“incalcolabile”.

Per riflettere sulla relazione tra l'uomo moderno e la tecnica, Heidegger sottolinea che è necessario riconoscere il nichilismo come l'evento che attraversa profondamente da sempre la storia culturale della metafisica in modo tale da confrontarsi compiutamente con siffatta storia nichilistica, lasciando emergere tutta la sua natura.

Cosicché, Hölderlin e il discorso sull'arte diventano fondamentali per ripensare (umdenken) oggi il nichilismo e per sviluppare una riflessione che ruoti attorno alla questione della tecnica moderna e che riesca, in un certo modo, a cogliere il fenomeno nichilistico nella sua massima problematicità, ricomprendendolo e insieme oltrepassandolo. In questa prospettiva, l'arte in quanto Dichtung offre a noi la possibilità di arrestarci dinanzi all'apparire delle cose, permettendo di riallacciare il dialogo interrotto con l'essere: nelle scarpe di van Gogh, nel tempio greco, nella poesia di Hölderlin la relazione originaria tra l'uomo e l'essere è, ogni volta, messa al riparo e mantenuta.

Se il nichilismo metafisico nella sua più compiuta forma tecnica non fa altro che sfruttare e prosciugare l'ente, ponendo l'accento sul suo funzionamento e sulla sua utilizzabilità, l'arte, nel suo significato più proprio, trascende piuttosto l'ambito dell'utilità e della riproducibilità tecnica, superando "ogni sconvolgimento dovuto al furore del calcolare"63, valorizzando l'ente nel suo pieno essere. È ciò che accade, ad esempio, nell'inno Der Rhein

$61 \mathrm{lb}$.

62 lb. p. 59.

63 HeidegGer, M. "Pensiero rimemorante" e "Mnemosyne", in Hölderlin. Viaggi in Grecia. Trad. T. Scappini. Milano, Bompiani, 2012, p. 19. 
di Hölderlin ${ }^{64}$. Cantando la peculiare natura sfuggente ed indomabile del fiume, il poeta ne esalta la purezza e l'incessante sgorgare. Questa natura è letteralmente enigmatica, dal momento che non può essere spiegata o compresa logicamente. Nella poesia, il Reno non assume affatto il significato di un oggetto "impiegato" (bestellte), uno strumento d'esame e di indagine, un mero dato oggettuale; viceversa, esso acquisisce la forma della parola in movimento che sfugge di conseguenza ad ogni intervento tecnico-produttivo, rimanendo inafferrabile giacché non si risolve mai in alcuna presenza concreta e determinata. Così, il fiume viene poetato e pensato essenzialmente come ciò che, nel suo inarrestabile fluire, mai si oggettiva in una definizione chiara e determinata, e perciò mai si entifica. Viceversa, esso è e rimane "un enigma che puro sgorga"65; un enigma da conservare e mantenere nella sua originaria indecifrabilità.

In questa maniera, Hölderlin viene descritto da Heidegger come il poeta che esperisce su di sé tutta la cifra ontologica del linguaggio poetico, articolando attraverso l'emergere di un pensiero poetante una riflessione sulla natura dei fenomeni, dicendola e mostrandola. Nella forza del suo canto poetico, Hölderlin "non dà alcuna dottrina e non pone alcuna legge. Non costruisce alcuna figura e non effettua alcuna descrizione" ${ }^{\prime 66}$. Ciononostante, i suoi versi ogni volta danno vita a nuove condizioni di possibilità e a nuovi orizzonti di senso segnati dalla presenza originaria dell'essere. Essi mettono in guardia dai pericoli che possono emergere da una rigorosa oggettivazione dell'essere. Ma è dove c'è la consapevolezza di questo pericolo che sorgono di continuo quelle condizioni essenziali grazie alle quali scorgere una via d'uscita, la salvezza al di là del pericolo ${ }^{67}$. È perciò Hölderlin "il poeta del poeta" ${ }^{68}$ che "nella fragilità del suo essere che non di rado si ripiega timorosamente in se stesso, conosce in modo perfettamente chiaro la peculiarità della poesia" ${ }^{\prime 2}$. I suoi versi, infine, designano il luogo, sconfinato e vastissimo, che si sottrae al

64 Cfr. Hölderlin, F. "Der Rhein. An Isaak von Sinclair", in Tutte le liriche. Op. cit. pp. 329-341.

65 La citazione è la traduzione italiana, curata da Reitani, del seguente verso hölderliniano: "Ein Rätsel ist Reinentsprungenes" (HöLDERLIN, F. "Der Rhein. An Isaak von Sinclair". Op. cit. v. 46).

66 HeIdegGeR, M. "Frammenti hölderliniani dell'Empedocle", in Hölderlin. Viaggi in Grecia. Op. cit. p. 633.

67 In questa maniera, Heidegger reinterpreta i celebri versi dell'inno Patmos di Hölderlin che così recitano in tedesco: "Wo aber Gefahr ist, wächst / Das Rettende auch" (HöLDERLIN, F. "Patmos", in Sämmtliche Werke, Bd. 2, hrsg. von F. Beissner. Stuttgart, Kohlhammer, 1951, s. 173. vv. 3-4); "Ma dove è il pericolo, germoglia / anche ciò che salva" (trad. mia). Con essi, il filosofo chiude il saggio Die Frage nach der Technik pubblicato nel 1953 presso l'editore Neske, tratto da una conferenza tenuta il 18 novembre dello stesso anno a Monaco presso l'Accademia Bavarese delle Belle Arti (oggi in Vorträge und Aufsätze. GA 7. Frankfurt am Main, Klostermann, 2000, pp. 7-36). Si veda a proposito Heidegger, M. "La questione della tecnica", in Saggi e discorsi, Op. cit. pp. 22 sgg.

68 Heidegger, M. "Hölderlin e l'essenza della poesia", in La poesia di Hölderlin. Op. cit. p. 42.

69 HeIDEgGer, M. "La poesia", in La poesia di Hölderlin. Op. cit. p. 220. 
dominio del nichilismo, lasciando vedere quella salvezza che significa il "ritorno a casa" (Heimkehr) dell'uomo, la riconquista del significato originario dell'essere.

\section{Riflessioni conclusive.}

Sulla base di quanto esposto, risultano ben visibili le implicazioni pratiche della speculazione filosofica heideggeriana. L'analisi sul malessere della contemporaneità consente ad Heidegger di osservare con occhi lucidi i più devastanti esiti della tecnicizzazione illimitata, echi di un lungo processo articolatosi nel tempo che ha radicalmente trasfigurato il volto della nostra epoca: la riduzione dell'essere ad ente, l'imporsi di una ragione strumentale, la costituzione di un sistema fondato sulla produzione e sulla funzionalità, la crisi delle ideologie tradizionali, lo sviluppo planetario del progresso tecnologico, l'irrefrenabile sfruttamento della terra.

Da questo punto di vista, la Seinsfrage può essere interpretata come il motivo ricorrente e fondamentale attraverso cui ricomprendere i problemi più emergenti e cruciali dell'attualità per ripensare, e in un certo senso ricreare, la relazione tra l'uomo e la natura. Cogliendo in tutta la sua radicalità l'aspetto totalizzante della tecnica moderna, Heidegger denuncia lo sfruttamento delle risorse terrestri operato dalla volontà di potenza umana, il cui risultato più immediato è il controllo totale sull'ambiente circostante che porta ad una sorta di snaturalizzazione dei fenomeni naturali. In questo processo, l'uomo ogni volta oltrepassa le proprie possibilità e i propri limiti, sconvolgendo di conseguenza i ritmi e gli equilibri della natura:

La legge nascosta della terra la mantiene nella moderata misuratezza del nascere e del perire di tutte le cose entro i limiti della loro possibilità, che ognuna di esse segue e che tuttavia nessuna conosce. La betulla non oltrepassa mai la sua possibilità. Il popolo delle api abita dentro all'ambito della sua possibilità. Solo la volontà, che si organizza, con la tecnica, in ogni direzione, fa violenza alla terra e la trascina nell'esaustione, nell'usura e nelle trasformazioni dell'artificiale. Essa obbliga la terra ad andare oltre il cerchio della possibilità che questa ha naturalmente sviluppato, verso ciò che non è più il solo possibile, e quindi è l'impossibile ${ }^{70}$.

L'insistenza sulla necessità di sviluppare una riflessione originaria sull'essere pone, in fondo, l'accento sul rapporto problematico dell'umanità con l'ambiente, offrendo altresì nuove possibilità di comprensione di noi stessi e del nostro pianeta. Le interrogazioni sulla

70 HeIDEgGeR, M. "Oltrepassamento della metafisica", in Saggi e discorsi. Op. cit. p. 64. 
Dichtung e sullo Andenken rappresentano, dunque, il tentativo da parte del filosofo di reagire agli squilibri e alle trasformazioni che colpiscono il profilo della natura; allo stesso tempo, suddette interrogazioni ci danno l'opportunità di riflettere sull'essere delle cose e dei fenomeni, ponendo le basi per la costruzione di un modello filosofico -alternativo a quello della razionalità scientifica tecnicizzante e depauperante- fondantesi sulla tesi secondo la quale la totalità della natura non si esaurisce affatto con l'uomo. Ciò significa da un alto pensare la natura non come mera materia inerte e passiva, oggettivabile e plasmabile a piacere; dall'altro, invece, vuole dire soffermarsi sui limiti e le possibilità di un essere umano non più signore o padrone della natura, bensì suo dimorante e pastore.

\section{Riferimenti bibliografici.}

ARIStotele. Etica Nicomachea. Trad. C. Mazzarelli. Milano, Bompiani, 2000.

BalazUt, J. Heidegger. Une philosophie de la présence. Paris, L’Harmattan, 2013.

BALAZUT, J. Heidegger et l'essence de la poésie. Paris, L'Harmattan, 2017.

BALAZUT, J. "La thèse de Heidegger sur l'art", en Nouvelle revue d'esthétique 5/1, 2010, pp. 141-152.

CURIA, M. II nichilismo e la tecnica. Tra Heidegger e Severino. Firenze, Clinamen, 2014.

DE ALESSI, F. Heidegger lettore dei poeti. Torino, Rosenberg \& Sellier, 1991.

DeRRIDA, J. Psyché. Invenzioni dell'altro. Vol. 2. Trad. R. Balzarotti. Milano, Jaka Book, 2009.

Dewalque, A. Heidegger et la questione de la chose. Paris, L'Harmattan, 2003.

FABRIS, A. "L'ermeneutica della fatticità' nei corsi friburghesi dal 1919 al 1923", in VolPI, F. (a cura di). Guida a Heidegger. Bari, Laterza, 1998.

GiAnnetTo, E. Sguardi sul pensiero contemporaneo. Filosofia e scienze per cambiare il mondo. Padova, Libreriauniversitaria.it, 2018.

HARMAN, G. Heidegger Explained. From Phenomenon to Thing. Chicago, Open Court, 2007.

HeIdegger, M. Che cosa significa pensare?. Trad. U. Ugazio e G. Vattimo. Milano, Sugarco, 1988.

Heidegger, M. Essere e tempo. Trad. A. Marini. Milano, Mondadori, 2013.

Heldegger, M. Gli inni di Hölderlin "Germania" e "Il Reno". Trad. G. B. Demarta. Milano, Bompiani, 2005. 
HeIdegger, M. Hölderlin. Viaggi in Grecia. Trad. T. Scappini. Milano, Bompiani, 2012.

Heidegger, M. In cammino verso il linguaggio. Trad. A. Caracciolo. Milano, Mursia, 2014. Heidegger, M. L'inno "Andenken" di Hölderlin. Trad. C. Sandrin e U. Ugazio, Milano, Mursia, 1997.

Heidegger, M. L'inno "Der Ister" di Hölderlin. Trad. C. Sandrin e U. Ugazio. Milano, Mursia, 2003.

Heidegger, M. L'origine dell'opera d'arte. Trad. G. Zaccaria e I. De Gennaro. Milano, Marinotti, 2012.

Heidegger, M. La poesia di Hölderlin. Trad. L. Amoroso. Milano, Adelphi, 2001.

HeIdegGeR, M. Lettera sull'"umanismo". Trad. F. Volpi. Milano, Adelphi, 2008.

Heidegger, M. Nietzsche. Trad. F. Volpi. Milano, Adelphi, 1994.

HeIdegger, M. Saggi e discorsi. Trad. G. Vattimo. Milano, Mursia, 2015.

Heidegger, M. Segnavia. Trad. F. Volpi. Milano, Adelphi, 2008.

Helting, H. "Alétheia-Etymologien vor Heidegger im Vergleich mit einigen Phasen der alétheia-Auslegung bei Heidegger", in Heidegger-Studien 13, 1997, pp. 91-107.

von Herrmann, F.-W. La filosofia dell'arte di Martin Heidegger. Un'interpretazione sistematica del saggio "L'origine dell'opera d'arte". Trad. I. De Gennaro e M. Amato. Milano, Marinotti, 2001.

HöLDERLIN, F. Le liriche. Trad. E. Mandruzzato. Milano, Adelphi, 1977.

Hölderlin, F. "Patmos", in Sämmtliche Werke, Bd. 2, hrsg. von F. Beissner. Stuttgart, Kohlhammer, 1951.

HölderlıN, F. Poesie. Trad. G. Vigolo. Milano, Mondadori, 1986.

HöLDERLIN, F. Tutte le liriche. Trad. L. Reitani. Milano, Mondadori, 2001.

KISIEL, T. The Genesis of Heidegger's "Being and Time". Berkeley, University of California Press, 1993.

KLUGE, F. Etymologisches Wörterbuch. Berlin, De Gruyter, 1960.

KoCKELMANS, J. J. Heidegger on art and art works. Dordrecht, Nijhoff, 1985.

MARAFIOTI, R. M. La questione dell'arte in Heidegger. Soveria Mannelli (CZ), Rubbettino, 2008. 
MorettI, G. Il poeta ferito. Hölderlin, Heidegger e la storia dell'essere. Imola, La Mandragora, 1999.

Panattoni, R.; Grazioli, E. (a cura di). Le scarpe di Van Gogh. Milano, Marcos y Marcos, 2013.

Platone. Simposio. Trad. G. Reale. Milano, Bompiani, 2000.

Platone. Sofista. Trad. B. Centrone. Torino, Einaudi, 2008.

PöggeleR, O. Neue Wege mit Heidegger. Freiburg, Alber, 1992.

Richardson, W. J. Heidegger. Through Phenomenology to Thought. The Hague, Nijhoff, 1963

RovattI, P. A. La posta in gioco. Heidegger, Husserl e il soggetto. Milano, Bompiani, 1987.

RuggenINI, M. (a cura di). Heidegger e la metafisica. Genova, Marietti, 1991.

SAFrAnskI, R. Heidegger e il suo tempo. Trad. M. Bonola. Milano, TEA, 2008.

Semerari, G. (a cura di). Confronti con Heidegger. Bari, Dedalo, 1992.

SeVerino, E. L'essenza del nichilismo. Milano, Adelphi, 1982.

SeVErino, E. Heidegger e la metafisica. Milano, Adelphi, 1994.

Sinnerbrink, R. "A Heideggerian Cinema?: On Terence Malick's The Thin Red Line", in FilmPhilosophy 10/3, 2006, pp. 26-37.

SoncINI, U. "La tematica del nichilismo in Nietzsche, Heidegger, Severino", in Divus Thomas 102, 1999, pp. 182-223.

Stambaugh, J. The Finitude of Being. Albany, State University of New York Press, 1992.

THURNHER, R. “iA las cosas mismas! Acerca de la significación de la máxima fenomenología fundamental en Husserl y Heidegger", en Seminarios de filosofía 9, 1996, pp. 24-44.

ToscanI, F. L'“azzurro della scuola degli occhi". Terra e cielo di Hölderlin e di Heidegger. Piateda (So), CFR, 2012.

ToscanI, F. Poesia e pensiero nel "tempo di privazione". In cammino con Hölderlin e Heidegger. Pistoia, Petite Plaisance, 2010.

Vattimo, G. Poesia e ontologia. Milano, Mursia, 1985.

Vitiello, V. Utopia del Nichilismo. Tra Nietzsche e Heidegger. Napoli, Guida, 1983.

VolPI, F. Il nichilismo. Bari, Laterza, 2009. 
VolPI, F. "'Noi senza patria': Heidegger e la Heimatlosigkeit dell'uomo moderno", en Revista Portuguesa de Filosofia 59, 2003, pp. 1261-1267.

WELSCH, W. La terra e l'opera d'arte. Heidegger e il Crepuscolo di Michelangelo. Trad. U. Ugazio. Ferrara, Gallio Editore, 1991.

Young, J. Heidegger's Philosophy of Art. Cambridge, Cambridge University Press, 2001. 